\title{
A DIFFERENTIAL STUDY ON THE FAVOURABLE NANOPARTICLE FORMULATION OF THE ANTIMALARIAL DRUG HALOFANTRINE
}

\author{
NOHA TALAL ZELAI* \\ Department of Biological Science, Faculty of Science, \\ King Abdulaziz University, Aljamea Road, Jeddah 21551, Saudi Arabia
}

\begin{abstract}
To tackle chloroquine-resistant Plasmodium parasites, the characteristics of conventional medicines should be improved. Halofantrine (HF) is one such successful drug that is used to treat malaria. However, it is poorly soluble, with non-dose-proportional bioavailability, and causes cardiac side effects. In this study, HF was formulated with five different nanoformulations: nanoprecipitation suspension with two different preparation protocols (NPSI and NPSII), emulsification/solvent evaporation by high shear homogenization (ESEhsh), emulsification/solvent evaporation by tip sonication (ESEts), and nanoliposomes (NLS). ESEts nanoparticles showed a size of $100 \pm 26 \mathrm{~nm}$ with fair homogeneity (polydispersity index $=0.3 \pm 0.04)$, reasonable encapsulation efficiency $(26 \pm 0.9 \%$ ), high anionic charge (zeta potential $=-76 \pm 3 \mathrm{mV})$, and slow-release $(62 \pm 7 \%)$ within $72 \mathrm{~h}$. Therefore, ESEts is a promising candidate for HF-based antimalarial drug formulations for in vivo models.
\end{abstract}

Keywords: halofantrine, malaria, nanoprecipitation, emulsification/ solvent evaporation, nanoliposomes

Halofantrine (HF) is a phenanthrene methanol antimalarial drug. It targets the schizont stage of the parasite and interferes with hemoglobin digestion. It is used to treat infections caused by both chloroquine-sensitive and resistant Plasmodium falciparum strains (1).

In a previous study, HF formulations were prepared either with poly(D,L-lactide) or with surfacemodified poly(D,L-lactide) nanoparticles. The yielded nanoparticles contained $95 \%$ HF of $180 \mathrm{~nm}$ size. HF nanoparticles doses higher than this were found to be safer than free HF on the hearts of animals (2). In another study, nanoparticles of an average size of $416 \pm 38 \mathrm{~nm}$ (PDI $=0.350$, zeta potential $=+10.9 \pm 1.2 \mathrm{mV}$ ), were generated from poly- $\varepsilon-$ caprolactone nanoprecipitation (3).

Unlike chloroquine, HF is not a rapidly absorbed drug. It is a lipophilic non-water-soluble antimalarial medicine. It is not given as a firstchoice medication due to its non-dose-proportional bioavailability (for $500 \mathrm{mg}$ or a higher dose) and its cardiac side effects $(4,5)$. Therefore, there is an urgent need to enhance the physicochemical properties of this drug using nanoformulations, to restore the use of this drug in chloroquine-resistant cases. Freebase HF is lipophilic, therefore, nanoprecipitation, nanoemulsion, and nanoliposomes seem to be great formulations in which oil is dispersed in water $(6,7)$.

This study aimed to develop a novel nanoformulation for HF with a smaller size, lower PDI, higher zeta potential, and better encapsulation efficiency. For this, HF formulations were prepared as nanoprecipitation suspension I (NPSI), nanoprecipitation suspension II (NPSII), emulsification/ solvent evaporation (by high shear homogenization, ESEhsh), emulsification/ solvent evaporation (by tip sonication, ESEts), and nanoliposomes (NLS). This study presents the comparative results of these five strategies of loading HF in nanocarriers.

\section{EXPERIMENTAL}

\section{Materials}

Halofantrine (HF, CAS:69756-53-2, reagent grade, purity $\geq 95 \%$ ) was purchased from LGM Pharma (Nashville, Tennessee, USA). Other reagents used in this study were chloroform, methanol, and dichloromethane (DCM) from Fisher Chemicals (Pittsburgh, Pennsylvania, USA), acetone from Tedia (Fairfield, Connecticut, USA), DMSO from Loba Chemie (Mumbai, India), Poly(lactide-co-Glycolide)

* Corresponding author: e-mail: nzelai@kau.edu.sa 
(PLGA) 50 : 50 (CAS: 26780-50-7; 24-38 kDa), dimethyl dioctadecyl ammonium bromide (DDA) $\geq 98 \%$, ethanol, cholesterol $\geq 99 \%$, and poloxamer 188 were from Sigma-Aldrich (Saint-Louis, Missouri, USA), lecithin from Acros Organics (CAS: 8002-43-5), and polyvinyl alcohol (PVA) was purchased from Spectrum (CAS 9002-89-5).

\section{Preparation of HF nanoparticles suspension I (NPSI) by nanoprecipitation}

Twenty milligrams of PLGA and $20 \mathrm{mg} \mathrm{HF}$ were dissolved in $2 \mathrm{~mL}$ of DMSO. This was then injected slowly in $50 \mathrm{~mL}$ of deionized water under high magnetic stirring. The resulting nanoparticles were left under stirring for $4 \mathrm{~h}$ in a wide mouth beaker and then evaporated in a rotary evaporator under reduced pressure (under $42 \mathrm{mbar}$ ) at $40^{\circ} \mathrm{C}$ to remove DMSO and deionized water. The final volume $(5 \mathrm{~mL})$ was centrifuged for $30 \mathrm{~min}$ at $65390 \mathrm{xg}$ at $10^{\circ} \mathrm{C}$. The supernatant was saved to examine the amount of $\mathrm{HF}$, while the nanoparticles in the base were used in all other experiments. For comparison, drugless PLGA nanoparticles were prepared using an earlier published protocol with modifications (8).

\section{Preparation of HF nanoparticles suspension II (NPSII) by nanoprecipitation}

Lecithin $(250 \mathrm{mg})$ was dissolved in ethyl alcohol: acetone mixture (1:3 ratio, total $25 \mathrm{~mL}$ ) placed in a wide mouth beaker using sonication and vigorous magnetic stirring (note: dissolution was not complete but led to fine suspended powder). Then, $50 \mathrm{mg}$ of PLGA and $50 \mathrm{mg}$ of HF, partially dissolved in $2 \mathrm{~mL}$ of acetone, were added to the beaker under stirring. The contents of this beaker were added dropwise slowly to the mixture of $250 \mathrm{mg}$ poloxamer dissolved in $50 \mathrm{~mL}$ of deionized water. This led to the formation of a translucent, and then heavy whitish nanoparticle suspension. The suspension was left overnight under high stirring and evaporated in a rotary evaporator under reduced pressure at $40^{\circ} \mathrm{C}$ to allow the evaporation of all organic solvents and hardening of the formed nanoparticles. On the next day, foam $(5 \mathrm{~mL})$, froth, and some aggregates were filtered using a 1-micron cellulose filter mesh, and nanoparticles passing through the filter were collected (8).

\section{Preparation of PLGA Nanoparticles by emulsification/solvent evaporation (ESE)}

Twenty milligrams of PLGA and $20 \mathrm{mg}$ of HF were dissolved in $5 \mathrm{~mL}$ of DCM. This was then added to $50 \mathrm{~mL}$ deionized water containing $4 \%$ PVA. Both were emulsified using either high shear homogenization for $3 \mathrm{~min}$ at $600 \mathrm{rpm}$ (ESEhsh) or sonicated with a tip sonicator (ESEts) for $40 \mathrm{~min}$ at $50 \%$ power. The resulting primary emulsion was added to $50 \mathrm{~mL}$ deionized water containing $0.5 \%$ PVA to hasten DCM escape and evaporation leading to the solidification of particles. Particles were left for $4 \mathrm{~h}$ under high magnetic stirring and evaporated under reduced pressure at $40^{\circ} \mathrm{C}(9)$. The final volume was nearly $5 \mathrm{~mL}$.

\section{Halofantrine (HF)-loaded nanoliposomes (NLS)}

Cationic nanoliposomes were prepared as previously described (10). In brief, a stock of $50 \mathrm{mg}$ DDA was prepared in $10 \mathrm{~mL}$ chloroform. Another stock of $50 \mathrm{mg}$ cholesterol was prepared in $10 \mathrm{~mL}$ chloroform. Twenty milligrams HF was dissolved in $20 \mathrm{~mL}$ chloroform. A 70\% of DDA stock of nanoliposomes, $30 \%$ of cholesterol stock, and $100 \%$ HF solution were prepared. Nanoliposomes mixture was dried under a stream of $\mathrm{N}_{2}$, then left in vacuum for $1 \mathrm{~h}$. The dried lipid film was rehydrated by $1 \mathrm{~mL}$ of normal saline and heated for $15 \mathrm{~min}$ at $60^{\circ} \mathrm{C}$, followed by sonication for $30 \mathrm{~min}$ at $60^{\circ} \mathrm{C}$. Contents of each vial were transferred to microfuge tubes (Eppendorf, Germany), individually, and centrifuged for $5 \mathrm{~min}$ at $10000 \mathrm{rpm}$ to exclude the non-encapsulated drug together with non-hydrated lipids.

\section{Measurement of particle size and zeta potential}

All samples were given similar treatments. One milliliter of the nanoformulation was added to $1 \mathrm{~mL}$ of deionized water, sonicated for $1 \mathrm{~min}$, and filtered through a one-micron cellulose filter mesh. Zetasizer and DTS1060C zeta cell (Malvern, UK) were used to analyze PDI, particle size, and zeta potential of the prepared, loaded nanoformulations. The apparatus was adjusted to a laser wavelength of $633 \mathrm{~nm}$ and a scattering angle of $173^{\circ}$.

\section{Measurement of drug content and release profile}

Three samples of HF-loaded nanoformulations (each $0.5 \mathrm{~mL}$ ) were first diluted in an appropriate solvent then mixed with phosphate-buffered saline (PBS) to prepare a theoretical concentration of $62.5 \mathrm{mg}$. Each sample of NPSI, NPSII, ESEhsh, and ESEts was diluted in $0.5 \mathrm{~mL}$ acetone to dissolve the polymer and free the drug, while each sample of NLS was dissolved in $0.5 \mathrm{~mL}$ of methanol to dissolve the lipids and release the drug. All samples were vigorously vortexed for $5 \mathrm{~min}$. In each vial, $0.9 \mathrm{~mL}$ of $\mathrm{HF}$ nanoformulation was mixed with $0.1 \mathrm{~mL}$ of losartan potassium (the internal standard). Samples were analyzed by HPLC, then the encapsulation efficiency 
was calculated by determining the UV reading of each sample.

HPLC analysis (Waters 2695 with 996 Photodiode Array Detector) was done following a previously published method with modifications (11). In brief, the wavelength was adjusted to $254 \mathrm{~nm}$. Methanol $/ 0.05 \mathrm{M}$ KH2PO4 (20: 80, v/v, pH 3.1) was used as a mobile phase. The analyte was separated using the $\mathrm{C} 18$ column $(5 \mu, 150 \mathrm{~mm} \times 4 \mathrm{~mm}$ i.d. $)$, in $30^{\circ} \mathrm{C}$ with a flow rate of $0.5 \mathrm{~mL} / \mathrm{min}$.

One milliliter of HF-loaded nanoformulation and $5 \mathrm{~mL}$ of PBS were placed in a dialysis bag (Spectra/Por membrane, molecular weight cut-off 6-8 kDa, Illkirch, France) at the bottom of a $500 \mathrm{~mL}$ glass beaker containing $250 \mathrm{~mL}$ PBS ( $\mathrm{pH} 7.4)$. The beaker was horizontally shaken $(80 \mathrm{rpm})$ in a thermostatic oven adjusted at $37^{\circ} \mathrm{C}$. At predetermined time intervals $(0,1,4,8,12,24,48$, and $72 \mathrm{~h}), 0.9 \mathrm{~mL}$ samples were withdrawn individually, in vials containing $0.1 \mathrm{~mL}$ of internal standard and analyzed through HPLC. All withdrawn samples were replaced with the fresh medium to maintain sink conditions.

\section{Statistical analysis}

All analyses were performed in triplicates and expressed as means \pm standard error of the mean (S.E.M). Encapsulation efficiency was calculated as the mean percentage \pm S.E.M.

All samples were assumed to have a Gaussian distribution. Experiments were designed with no matching or pairing because each formulation was prepared separately. One-way analysis of variance (ANOVA) was used to determine significant differences between groups.

Tukey's multiple comparisons test was used to determine whether the two groups were significantly different.

When P-value was lower than 0.05 , the difference was considered statistically significant.

\section{RESULTS}

\section{Average size, polydispersity index (PDI), and zeta potential}

Five different formulations of HF nanoparticles were prepared and were assessed for the most optimum physical properties. All estimations of particle size, PDI, encapsulation efficiency, and zeta potential for nanoformulations are summarized in Table 1.

A significant difference in particle size and PDI among all formulations was observed through ANOVA. Surprisingly, there was no statistical difference between the average size of nanoparticles in NPSI and blank nanoprecipitation (BNS) ( $\mathrm{P}>0.9999$ for particle size) and $(\mathrm{P}=0.7414$ for $\mathrm{PDI})$, as the average size of NPSI was $124.7 \pm 2.3 \mathrm{~nm}$, with PDI of $0.149 \pm 0.006$ and the average size of BNS was $112 \pm 5.6 \mathrm{~nm}(\mathrm{PDI}=0.285 \pm 0.05)$.

From NPSII, small nanoparticles with a size of $140 \pm 34 \mathrm{~nm}$ were obtained which was not significantly different from NPSI nanoparticles.

Zeta potential was found to be negative with an average value of $-12.5 \pm 1.6,-17.07 \pm 1.02,-17.08 \pm 1.1$, $-15.7 \pm 1.4$, and $-76 \pm 3 \mathrm{mV}$, for BNS, NPSI, NPSII, ESEhsh, and ESEts, respectively (P-value for all nanoformulations $=0.1205$ ). However, nanoliposomes exhibited a highly positive zeta potential $(33.6 \pm 1.1 \mathrm{mV})$.

\section{Encapsulation efficiency and release profile}

Upon estimating the free drug, $100 \%$ of added HF was found in the supernatant by using the NPSI, indicating that the encapsulation efficiency was $0 \%$. Similarly, NPSII resulted in $2.1 \pm 0.7 \%$ encapsulation efficiency $(\mathrm{P}=0.8984)$.

The loading capacity of nanoparticles prepared by emulsification/ solvent evaporation with high sheer homogenization was similar $(\mathrm{P}=0.4890)$ to that prepared with tip sonication $(20 \pm 2.3 \%, 26 \pm 0.9 \%$,

Table 1. Average size, polydispersity index (PDI), encapsulation efficiency, and zeta potential of the nanoformulations.

\begin{tabular}{|c|c|c|c|c|}
\hline Method & $\begin{array}{l}\text { Average size } \\
\quad(\mathrm{nm})\end{array}$ & PDI & $\begin{array}{l}\text { Zeta potential } \\
\qquad(\mathrm{mV})\end{array}$ & $\begin{array}{l}\text { Encapsulation } \\
\text { efficacy }(\%)\end{array}$ \\
\hline Blank Nanoprecipitation Solution (BNS) & $112 \pm 5.6$ & $0.285 \pm 0.05$ & $-12.5 \pm 1.6$ & - \\
\hline Nanoprecipitation suspension I (NPSI) & $124.7 \pm 2.3$ & $0.149 \pm 0.006$ & $-17.07 \pm 1.02$ & 0 \\
\hline Nanoprecipitation suspension II (NPSII) & $140 \pm 34$ & $0.23 \pm 0.02$ & $-17.08 \pm 1.1$ & $2.1 \pm 0.7$ \\
\hline $\begin{array}{l}\text { Emulsification / solvent evaporation } \\
\text { by high sheer homogenization (ESEhsh) }\end{array}$ & $495 \pm 165$ & $1 \pm 0.06$ & $-15.7 \pm 1.4$ & $20 \pm 2.3$ \\
\hline $\begin{array}{l}\text { Emulsification / solvent evaporation } \\
\text { by tip sonication (ESEts) }\end{array}$ & $100 \pm 26$ & $0.3 \pm 0.04$ & $-76 \pm 3$ & $26 \pm 0.9$ \\
\hline Nanoliposomes (NLS) & $348 \pm 23.3$ & $0.47 \pm 0.02$ & $33.6 \pm 1.1$ & $78.7 \pm 2.5$ \\
\hline
\end{tabular}




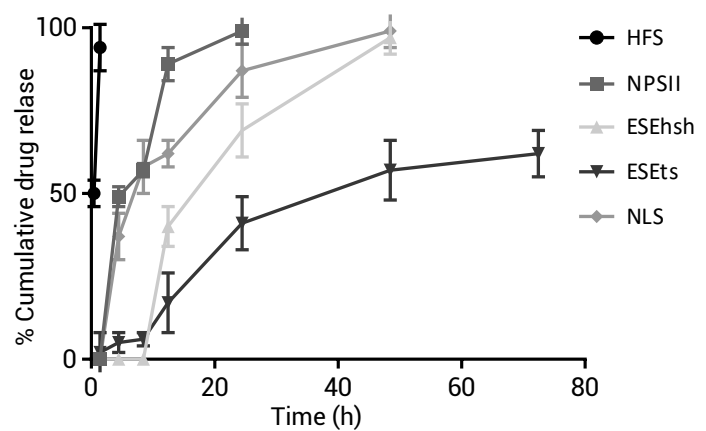

Figure 1. The release profile of the nanoformulations $(n=3)$. A high release profile was observed in the HF solution, where the release from NLS, ESEhsh, and ESEts was slower. Halofantrine solution (HFS); nanoprecipitation suspension II (NPSII); emulsification/ solvent evaporation by high shear homogenization (ESEhsh); emulsification/ solvent evaporation by tip sonication (ESEts); nanoliposomes (NLS).

respectively). The average encapsulation efficiency of NLS was $78.7 \pm 2.5 \%$, which was significantly higher than that of NPSI, NPSII, ESEhsh, and ESEts $(\mathrm{P}<0.0001)$.

The fastest release was observed in the HF solution as all $\mathrm{HF}$ was released within $1 \mathrm{~h}$ (P-value for all nanoformulations within 0 and $1 \mathrm{~h}<0.0001)$. After $4 \mathrm{~h}, 49 \pm 3 \%$ of HF was released from NPSII and $37 \pm 7 \%$ was released from NLS, while only $5 \pm 3 \%$ was released from ESEts and no HF was released from ESEhsh (P-value for all nanoformulations in $4 \mathrm{~h}<0.0001)$. The release of HF from ESEhsh started within $12 \mathrm{~h}(40 \pm 6 \%)$ and the drug release was complete by $48 \mathrm{~h}(97 \pm 5 \%)$. This release was comparable to that of NLS at 12, 24 and $48 \mathrm{~h}(\mathrm{P}=0.1391,0.3544$, 0.9752 , respectively). The release profile from ESEts increased steadily from $2 \pm 6 \%$ within $1 \mathrm{~h}$ to $62 \pm 7 \%$ at $72 \mathrm{~h}$. This was different from the drug release from NLS within $4,8,12,24$, and $48 \mathrm{~h}(\mathrm{P}=0.0025$, $0.0001,0.0043,0.0085$, and 0.0098 , respectively) as illustrated in Figure1.

\section{DISCUSSION AND CONCLUSION}

In this work, HF nanoformulations were prepared using five different methods and the most suitable formulation was evaluated to target the cells invaded by Plasmodium parasites. PLGA was used for all polymeric nanoformulations because it is a biodegradable polymer, can encapsulate both hydrophilic and hydrophobic drugs, and maintains sustained release of drugs $(12,13)$.

Narrow size distribution was obtained by the NPSII method as evidenced by a low PDI of $0.23 \pm 0.02$. In another study, nanocapsules with a larger size $(416 \pm 38 \mathrm{~nm}$, PDI of 0.35$)$ were obtained using HF nanoprecipitated poly- $\varepsilon$-caprolactone (3).
PLGA NPSI was stable without the addition of any stabilizer due to their inherent charge. The used PLGA was - $\mathrm{COOH}$ terminal, which ionized in water to $-\mathrm{COO}^{-}$causing repulsion between particles and preventing their aggregation.

Poloxamer 188 has significantly affected the reduction in the particle size (14), although NPSII and NPSI had similar particle sizes.

NPSI suspension failed to encapsulate HF. This was attributed to the use of DMSO. The added amount of DMSO acted as a cosolvent and caused the dissolution of HF in water, thus decreasing the tendency of HF to remain within PLGA during polymer solidification. This was proved by the blank nanoprecipitation suspension which showed similar size, PDI, and zeta potential. Similarly, NPSII also showed a poor encapsulation efficiency $(2.1 \pm 0.7 \%)$. Thus, nanoparticles generated from the nanoprecipitation method do not have sufficient properties to be admitted as a method to trap HF.

Nearly $80 \%$ of encapsulated drugs were found to release from PLGA nanoformulations within $24 \mathrm{~h}$ (15). In addition, commercial HF tablets and different microsphere lipid formulations have a rapid release profile within $7 \mathrm{~h}$ (16). Moreover, a rapid release may be associated with poor encapsulation and the leakage of nanoparticles by undissolved drug particles present near the surface. These factors illustrated relatively fast drug release (maximum $48 \mathrm{~h}$ ), in this study, from lipid-containing-nanoliposomes, PLGA nanoformulations, and the suspension of poorly encapsulated nanoparticles prepared by nanoprecipitation. On the other hand, nanoemulsions prepared by tip sonication have smaller particles with long-term stability (17), and this agreed with the small size and stability of ESEts that exhibited extended-release compared to those of other nanoformulations in the current study.

In addition to the high loading efficiency, nanoliposomes of a hydrophobic drug such as HF may be delivered to the target cell by the fusion of the lipid bilayer of liposomes with the lipid bilayer of the cell membrane (18).

ESEts has a large size $(100 \pm 26 \mathrm{~nm})$, better loading efficiency $(26 \pm 0.9 \%)$, and highly negative zeta potential $(-76 \pm 3 \mathrm{mV})$, because of the significant role of sonication in particle size reduction $(19,20)$. Moreover, these small particles exhibit a slow release of $62 \pm 7 \%$ within $72 \mathrm{~h}$.

High zeta potential has a remarkable effect on the formulation stability, attributable to the strong repulsion between similar surface charges in the suspension. In addition, HF ESEts nanoparticles are considered as strong anionic particles $(-76 \pm 3 \mathrm{mV}<-30)$. 
The anionic nanoparticles have a higher cell membrane permeability and have fewer toxic effects than those exerted by cationic nanoparticles (21).

\section{CONCLUSION}

We compared the physical characteristics and the release profile of five different methods of preparing HF nanoparticles and excluded NPSI and NPSII because of their low loading efficiency. Although nanoliposomes prepared in this study showed promising properties, emulsification/solvent evaporation with tip sonication was found to be the method of choice as it meets the needs of HF nanoparticle formation.

\section{Conflicts of interest}

The authors declare no conflict of interest.

\section{REFERENCES}

1. Ibezim E., Odo U.: Afr. J. Biotechnol. 7, 349 (2008).

2. Mosqueira V.C., Loiseau P.M., Bories C., Legrand P., Devissaguet J.P., Barrat G.: Antimicrob. Agents Chemother. 48, 1222 (2004).

3. Leite E.A., Grabe-Guimaraes A., Guimaraes H.N., Machado-Coelho G.L.L., Barratt G., Mosqueira V.: Life Sci. 80, 1327 (2007).

4. Moffat A.C., Osselton M.D., Widdop B., Watts J.O.: Clarke's analysis of drugs and poisons. 4th ed., pp. 1746-1747, Pharmaceutical Press, London 2011.

5. Karbwang J., Bangchang K.N.: Clin. Pharmacokinet. 27, 104 (1994).
6. Kumar S., Dilbaghi N., Saharan R., Bhanjana G.: BioNanoSci. 2, 227 (2012).

7. Wais U., Jackson A.W., He T., Zhang H.: Nanoscale 8, 1746 (2016).

8. Fessi H., Puisieux F., Devissaguet J.P., Ammour N., Benita S.: Int. J. Pharm. 55, R1-R4 (1989).

9. Surolia R., Pachauri M., Ghosh P.C.: J. Biomed. Nanotechnol. 8, 172 (2012).

10. Benatti C.R., Epand R.M., Lamy M.T.: Chem. Phys. Lipids. 145, 27 (2007).

11. Kolade Y.T., Babalola C.P., Scriba G.: J. Pharm. Biomed. Anal. 41, 315 (2006).

12. Ramazani F., Chen W., van Nostrum C.F., Storm G., Kiessling F., et al.: Int. J. Pharm. 499, 358 (2016).

13. Astete C.E., Sabliov C.M.: J. Biomater. Sci. Polym. Ed. 17, 247 (2006).

14. Sharma N., Madan P., Lin S.: Asian J. Pharm. Sci. 11, 404 (2016).

15. Niwa T., Takeuchi H., Hino T., Kunou N., Kawashima Y.: J. Control. Release 25, 89 (1993).

16. Attama A.A, Igbonekwu C.N.: Asian Pac. J. Trop. Med. 4, 253 (2011).

17. Kozlowski M.: Polymeric Nanomaterials for Nanotherapeutics. Vasile C. Ed., 1 st Edition, p. 1452, Elsevier 2018.

18. Youngren S.R., Mulik R., Jun B., Hoffmann P.R., Morris K.R., Chougule M.B.: AAPS Pharm. Sci. Tech. 14, 1012 (2013).

19. Nickel C., Angelstorf J., Bienert R., Burkart C., Gabsch S., et al.: J. Nanopart. Res. 16, 1 (2014).

20. Pradhan S., Hedberg J., Blomberg E., Wold S., Odnevall Wallinder I.: J. Nanopart. Res. 18, 285 (2016).

21. Clogston J.D., Patri A.K.: Methods Mol. Biol. 697, 63 (2011). 